\title{
Tromboprofilaxis en el paciente médico hospitalizado por medicina interna Un estudio de corte transversal
}

\author{
Thromboprophylaxis in the hospitalized medical \\ patient by internal medicine \\ A cross-sectional study
}

\author{
Juan José Diaztagle, Javier Enrique Cely, Jorge de Jesús Sánchez, \\ Fernando Antonio Sánchez, Elkin José Mendoza • \\ BogotÁ, D.C. (ColombiA)
}

\section{Resumen}

Introducción: el tromboembolismo venoso es una de las principales causas de morbimortalidad prevenible seguida de una hospitalización. Las heparinas han demostrado ser eficaces para su prevención, sin embargo se ha documentado la subutilización de estos fármacos, por lo que implementar medidas que garanticen la formulación adecuada es fundamental. En nuestra institución se han instaurado estrategias para mejorar la formulación de estos fármacos con resultados iniciales favorables, pero se desconoce el efecto a más largo plazo.

Métodos: estudio descriptivo, retrospectivo de corte transversal. Se evaluaron pacientes mayores de 18 años, hospitalizados por medicina interna entre junio y noviembre de 2012. Se estimó una muestra representativa de 102 pacientes. Se identificó la formulación de la tromboprofilaxis al segundo día de hospitalización, se determinó si fue adecuada según las guías institucionales y los errores en la prescripción de la misma. Se compararon los resultados con dos mediciones previas realizadas en la institución.

Resultados: de los 102 pacientes evaluados, la tromboprofilaxis fue adecuada en $63(61,8 \%)$ e inadecuada en 39 (38.2\%). Las causas más frecuentes de error fueron: formulación en pacientes de bajo riesgo $18(46.1 \%)$ y error por omisión en 12 (30.7\%) pacientes. La formulación en pacientes con indicación y sin contraindicación disminuyó de $92-82 \%$ y en pacientes sin indicación aumentó de 50-56.2\%, con relación a una medida previa realizada después de la difusión de guías institucionales.

Conclusiones: la tromboprofilaxis en pacientes hospitalizados por medicina interna en nuestra institución se ordena en un alto porcentaje, sin embargo debe ser mejorada. El principal error es la formulación en pacientes con riesgo bajo. La implementación de estrategias para mejorar la tromboprofilaxis logró una mejoría inicial, pero tiende a disminuir con el tiempo. Se requiere un trabajo continuado de múltiples medidas que garanticen su impacto favorable a largo plazo. (Acta Med Colomb 2015; 40: 227-233).

Palabras clave: tromboprofilaxis, tromboembolismo venoso, heparinas, prevención, adherencia.

\section{Abstract}

Introduction: venous thromboembolism is one of the major causes of preventable morbidity and mortality following a hospitalization. Heparins have proven effective for prevention; however, underutilization of these drugs has been documented, so that the implementation of measures to ensure the proper formulation is essential. Strategies to improve the formulation of these drugs have been established in our institution with favorable initial results, but the longer-term effect is unknown.

Methods: descriptive, retrospective cross-sectional study. Patients over 18 years hospitalized for internal medicine between June and November 2012 were evaluated. A representative sample of 102 patients was estimated. Thromboprophylaxis formulation was identified on the second day of hospitalization and it was determined if its prescription was appropriate in accordance with the
Dr. Juan José Diaztagle Fernández: Internista, Epidemiólogo, Magíster en Fisiología. Instructor de Medicina Interna, Fundación Universitaria de Ciencias de la Salud-Hospital de San José. Profesor Asociado, Departamento de Ciencias Fisiológicas, Universidad Nacional de Colombia; Dr. Javier Enrique Cely Delgado: Residente de Tercer Año de Medicina Interna, Fundación Universitaria de Ciencias de la Salud-Hospital de San José; Dr. Jorge de Jesús Sánchez Cantillo: Residente de Tercer Año de Medicina Interna, Fundación Universitaria de Ciencias de la Salud-Hospital de San José; Dr. Fernando Antonio Sánchez Esquivel: Residente de Tercer Año de Medicina Interna, Fundación Universitaria de Ciencias de la Salud-Hospital de San José; Dr. Elkin José Mendoza Olmos: Residente de Tercer Año de Medicina Interna Fundación Universitaria de Ciencias de la Salud-Hospital de San José. Bogotá, D.C. (Colombia).

Correspondencia. Dr. Juan José Diaztagle Fernández. Bogotá, D.C. (Colombia).

E-mail: juanchotagle@yahoo.es

Recibido: 21/VII/2015 Aceptado: 4/IX/2015 
institutional guidelines as well as errors in its prescription. The results were compared with two previous measurements realized in the institution.

Results: Of the 102 patients evaluated, thromboprophylaxis was adequate in $63(61.8 \%)$ and inadequate in $39(38.2 \%)$. The most frequent causes of error were: prescription in low-risk patients $18(46.1 \%)$ and error of omission in $12(30.7 \%)$ patients. The formulation in patients with an indication and without contraindication decreased from $92 \%$ to $82 \%$ and in patients with no indication increased from $50 \%$ to $56.2 \%$, compared to a previous measurement made after the dissemination of institutional guidelines.

Conclusions: thromboprophylaxis in hospitalized patients in internal medicine at our institution is organized in a high percentage, but must be improved. The main error is the formulation in patients with low risk. The implementation of strategies to improve thromboprophylaxis achieved initial improvement, but tends to decrease over time. Continued work of multiple measures to ensure their favorable long-term impact is required. (Acta Med Colomb 2015; 40: 227-233).

Keywords: thromboprophylaxis, venous thromboembolism, heparin, prevention, adhesion.

\section{Introducción}

El tromboembolismo venoso (TEV) es una de las principales causas de morbimortalidad prevenible durante la hospitalización. Cerca de un tercio de todos los casos ocurren en los tres meses siguientes a una hospitalización y ésta puede explicar hasta $10 \%$ de la mortalidad intrahospitalaria (1). Se ha demostrado que la administración de fármacos antitrombóticos, principalmente las heparinas, en pacientes con factores de riesgo para TEV son eficaces en su prevención. Sin embargo, solo 39.5-64\% de estos pacientes reciben alguna forma de profilaxis $(2,3)$.

Frente a los críticos hallazgos mencionados, implementar estrategias para mejorar la formulación de la tromboprofilaxis se ha convertido en tema que cobra importancia a nivel mundial (4-7), a lo cual no ha sido ajeno nuestra institución, en donde un equipo multidisciplinario liderado por el servicio de hematología estructuró un programa integral de mejoramiento en este tema. Dentro de los planes ejecutados se divulgaron afiches y guías plegables de bolsillo en donde se establece una escala de estratificación del riesgo basada en el II Consenso Colombiano de Tromboembolismo Venoso (8) y se dan unas directrices para la formulación adecuada de la tromboprofilaxis. La tromboprofilaxis se convirtió en una política institucional de seguridad del paciente y para el año 2011 la guía de tromboprofilaxis fue nombrada "Guía trazadora" de nuestro hospital (9).

Una medición realizada luego de llevar a cabo estas medidas demostró una mejoría en la formulación adecuada de la tromboprofilaxis. Sin embargo, la evaluación del éxito de las estrategias de implementación de guía a largo plazo no está bien documentada. Se ha demostrado como la implementación de éstas puede tener dificultades en su aplicación pasado cierto tiempo e incluso el éxito alcanzado inicialmente disminuye una vez se retira la medida $(10,11)$, por lo que su evaluación a largo plazo es fundamental.

Por tal motivo el objetivo de este estudio es realizar una nueva evaluación de la práctica de tromboprofilaxis en pacientes hospitalizados por el servicio de medicina interna, buscando evaluar si la mejoría alcanzada en la formulación de la tromboprofilaxis se mantuvo en el tiempo. Se pretende, además, describir la población de pacientes y los factores de riesgo para TEV, con el ánimo de plantear en el futuro estrategias complementarias a las ya establecidas de tal forma que se pueda garantizar una adecuada tromboprofilaxis en estos pacientes.

\section{Material y métodos}

Se realizó un estudio descriptivo, retrospectivo de corte transversal entre los meses de junio a noviembre de 2012 , donde se evaluó la tromboprofilaxis a las 48 horas de ingreso. Se incluyeron pacientes mayores de 18 años de edad hospitalizados por el servicio de medicina interna, excluyendo aquellos con indicación a su ingreso de anticoagulación plena o trombolisis o que tuvieran como impresión diagnóstica TEV. También se excluyeron pacientes con estancia hospitalaria menor a 48 horas.

Para definir el tamaño de muestra, se tuvo en cuenta un estimativo aproximado de 1202 egresos durante el periodo de tiempo por evaluar. Teniendo en cuenta un error tipo I del 5\%, prevalencia esperada de profilaxis adecuada en la población de $83 \%$ (obtenida en mediciones previas) (9) y una diferencia máxima esperada de 7\%, se estimó 102 pacientes como una muestra representativa de los egresos.

Para elegir los pacientes se realizó una selección aleatoria simple de los días comprendidos entre el 1 de junio y el 31 de noviembre de 2012 por el método coordinado negativo. El departamento de estadística del hospital entregó un listado del total de pacientes egresados por medicina interna en los días seleccionados. Seguidamente se solicitaron las historias clínicas en el archivo del hospital y se verificaron criterios de inclusión y exclusión. Se utilizó un formato de recolección en donde se anotaron datos de identificación, grupo etáreo, variables relacionadas como factor de riesgo para tromboembolia venosa o sangrado, la condición al egreso del hospital (vivo o muerto) y el tipo de fármaco o medida antitrombótica utilizado durante la hospitalización. 
Las guías institucionales del hospital, basadas en el II Consenso Colombiano de Tromboembolismo Venoso para la tromboprofilaxis, establece que el paciente médico puede estratificarse como alto o bajo riesgo de dos maneras, la primera de ellas según el diagnóstico de ingreso, donde cualquiera de las siguientes condiciones clasifica a los pacientes como alto riesgo: insuficiencia cardiaca, infección sistémica, neumonía, accidente cerebrovascular (ACV) con paresia, enfermedad pulmonar obstructiva crónica (EPOC) exacerbada, enfermedad neoplásica, enfermedad inflamatoria intestinal, estados hipercoagulables sin tratamiento, artritis reumatoide, lupus, síndrome nefrótico o antecedente de TEV. La segunda forma es mediante una escala que consta de dos pasos. El paso uno consiste en determinar si existe un factor de riesgo agudo para desarrollar TEV, y el paso dos en evaluar si existen condiciones de base del paciente que sean factores de riesgo para desarrollar TEV. Cada paso contiene una serie de ítems al cual se le asigna una puntuación, y si al sumar los puntos del paso uno y dos resulta mayor o igual a cinco, el paciente se clasifica como alto riesgo y se debe iniciar tromboprofilaxis (9). Para los casos de difícil decisión del riesgo tromboembólico se realizó un consenso de tres investigadores, uno de ellos el investigador principal, con el objetivo de disminuir el margen de mala estratificación de los pacientes.

Si el paciente se clasificaba como de alto riesgo se recomendaba dalteparina 5000 UI SC cada día, enoxaparina 40 $\mathrm{mg} \mathrm{SC/día,} \mathrm{nadroparina} 40 \mathrm{mg} \mathrm{SC/día,} \mathrm{fondaparinux} 2.5$ $\mathrm{mg} /$ día (se prefiere en pacientes con contraindicación para administrar heparinas de bajo peso molecular) o heparina no fraccionada 5000 UI SC c/8horas. Este último es preferible en caso de insuficiencia renal con tasa de filtración glomerular menor a $30 \mathrm{~mL} / \mathrm{min}$.

Tomando en cuenta el modelo anterior, se determinó si la profilaxis en los pacientes fue adecuada o inadecuada. Si el paciente recibió una profilaxis inadecuada se identificó cualesquiera de los cinco escenarios posibles de error en la formulación:

- Error por omisión: no se formuló tromboprofilaxis en pacientes con alto riesgo para TEV y en quienes no estaba contraindicado.

- Error por dosis inadecuada: se formuló tromboprofilaxis en pacientes con alto riesgo para TEV, pero la dosis del fármaco no es adecuada, ya sea por sobredosificación o dosis subterapéutica.

- Error por formulación en pacientes con riesgo bajo: se formuló tromboprofilaxis en paciente con bajo riesgo para TEV.

- Error por formulación en pacientes con alto riesgo de sangrado: se formuló tromboprofilaxis farmacológica en aquellos pacientes con sangrado o alto riesgo para desarrollar sangrado. Idealmente se recomienda para estos casos tromboprofilaxis mecánica.

- Error por mala elección del fármaco: se formuló tromboprofilaxis en pacientes con alto riesgo para
TEV, sin embargo el fármaco no es el apropiado para el paciente (Pej, administrar heparinas de bajo peso molecular a pacientes con enfermedad renal crónica y tasa de filtración glomerular menor a $30 \mathrm{~mL} / \mathrm{min}$ ).

Se compararon los resultados con dos mediciones previas de formulación de profilaxis antitrombótica en la institución. La primera fue realizada entre abril-agosto de 2009 y la segunda, posterior a la implementación de estrategias para mejorar la tromboprofilaxis entre agosto 2011 y mayo 2012. La comparación se realizó en las siguientes condiciones: Porcentaje de pacientes con riesgo de TEV en quien se formuló la profilaxis farmacológica (sin contraindicaciones) y porcentaje de pacientes con bajo riesgo para TEV (sin indicación para profilaxis) en quienes se formuló la tromboprofilaxis.

Se diseñó una base de datos en Microsoft Excel 2013 para almacenar la información de la población en estudio. El investigador principal se encargó de verificar la información de $10 \%$ de los formatos de recolección para garantizar la calidad de la información recolectada. El análisis estadístico se realizó con el programa STATA12. Las variables categóricas se presentan en proporciones y las cuantitativas se resumen con promedios y desviaciones estándar. Se describe la frecuencia de formulación adecuada de la tromboprofilaxis, medicamento formulado y el porcentaje de error en la formulación categorizado por cualquiera de los cinco escenarios ya descritos previamente.

El protocolo fue presentado y aprobado por el Comité de Investigaciones de la Facultad de Medicina de la Fundación Universitaria de Ciencias de la Salud, Hospital de San José de Bogotá y el Comité de Ética en Investigación para Humanos del Hospital de San José de Bogotá. Se consideró como una "investigación sin riesgo" por lo tanto no requirió consentimiento informado.

\section{Resultados}

Se tamizaron 163 pacientes, de los cuales se excluyeron 61 (36 por anticoagulación al ingreso, 13 por hospitalización menor de 48 horas, nueve por hospitalización por otros servicios, dos fueron menor de 18 años y uno ya se había incluido). Por lo tanto se evaluaron 102 pacientes, de los cuales 43 (42.2\%) fueron hombres, el promedio de edad fue 61.1 años $( \pm 19.76)$. La Tabla 1 describe las características generales de la población. No se reportaron pacientes con antecedentes de enfermedad inflamatoria intestinal, sangrado mayor, trombofilia, úlcera gástrica activa, insuficiencia hepática, posparto, cirugía mayor, trauma reciente o infarto. Entre los factores de riesgo más frecuentes encontrados están: movilidad reducida en $96(94.1 \%)$ pacientes, neoplasia activa en 17 (16.7\%), antecedente de EPOC en 23 (22.5\%) y de falla cardiaca en $10(9.8 \%)$ (Tabla 1$)$.

Los diagnósticos principales de ingreso más frecuentes fueron: neumonía en 20 pacientes (19.6\%), infección de vías urinarias en $16(15.7 \%)$ e insuficiencia cardiaca en 12 $(11.8 \%)$ (Tabla 2$)$. Se registraron cuatro (3.9\%) fallecidos, 
Tabla 1. Características demográficas de la población.

\begin{tabular}{|l|c|}
\hline \multicolumn{1}{|c|}{ Variable } & Media \\
\hline Género masculino n (\%) & $43(42.2)$ \\
Edad & $61.1( \pm 19.76)$ \\
\hline Medidas antropométricas & \\
Peso & $65.53( \pm 19.761)$ \\
Talla & $1.60( \pm 82)$ \\
IMC & $25.16( \pm 4.65)$ \\
\hline Paraclínicos & \\
Creatinina sérica & $1.18( \pm 1.11)$ \\
Tasa de filtración glomerular (mL/min/1.72m²) & $85.1( \pm 44.9)$ \\
Recuento plaquetario & $22068( \pm 125388)$ \\
\hline Datos de la hospitalización & \\
Estancia hospitalaria en días & $7.49( \pm 5.77)$ \\
Muerte al egreso n (\%) & $4(3.9)$ \\
\hline Comorbilidades y antecedentes n (\%) & $12(11.8)$ \\
Hipertensión arterial & $10(9.8)$ \\
Diabetes mellitus tipo 2 & $5(4.9)$ \\
Enfermedad pulmonar obstructiva crónica & $4(3.9)$ \\
Neoplasia activa & $3(2.9)$ \\
Enfermedad renal crónica & $1(0.9)$ \\
Tabaquismo & $25(24.5)$ \\
Obesidad & $17(16.7)$ \\
Insuficiencia cardiaca & $14.7)$ \\
Enfermedad reumatológica & $13.7)$ \\
ACV isquémico & \\
Tromboembolia venosa previa & \\
Androcedente de terapia de reemplazo hormonal & \\
\hline
\end{tabular}

y el promedio de estancia fue 7.4 días $( \pm 5.77)$. Setenta pacientes $(68.6 \%)$ fueron clasificados como alto riesgo para tromboembolia venosa, de los cuales 47 (67.2\%) cumplieron el criterio tanto por puntaje como por diagnóstico, 8 (11.4\%) por la escala de puntaje y 15 (21.4\%) por el diagnóstico de ingreso (Tabla 3).

\section{Uso de tromboprofilaxis}

Entre los 70 pacientes con indicación de profilaxis, tres (4.2\%) tuvieron contraindicación para profilaxis farmacológica por plaquetas menor a 50000 . De los 67 pacientes con indicación y sin contraindicación, 55 (82\%) fueron formulados con heparinas y de éstos la formulación fue adecuada en 49 (89\%) pacientes. Entre los 32 pacientes (31.3\%) con bajo riesgo para TEV 18 (56.2\%) tuvieron formulación de tromboprofilaxis (Figura 1).

Entre el total de pacientes evaluados, $63(61.8 \%)$ tuvieron un adecuado uso de la tromboprofilaxis, mientras que en $39(38.2 \%)$ la tromboprofilaxis fue inadecuada. Las causas de uso inadecuado fueron la formulación de profilaxis en pacientes con bajo riesgo en 18 (46.1\%) casos y el error por omisión en $12(30.7 \%)$. En un paciente se formuló tromboprofilaxis farmacológica aunque el paciente tenía bajo riesgo para TEV y además alto riesgo de sangrado. Entre los pacientes con indicación de profilaxis y contraindicación
Tabla 2. Diagnósticos de ingreso.

\begin{tabular}{|l|c|}
\hline \multicolumn{1}{|c|}{ Diagnóstico } & $\mathbf{n}(\%)$ \\
\hline Neumonía & $20(19.6)$ \\
Infección de vías urinarias & $16(15.7)$ \\
Insuficiencia cardiaca & $12(11.8)$ \\
Enfermedades pulmonares crónicas (EPOC-EPID-Asma) & $10(9.8)$ \\
Infecciones y otras alteraciones gastrointestinales & $9(8.8)$ \\
Enfermedad hematooncológica y sus complicaciones & $9(8.8)$ \\
Infección de tejidos blandos y otras infecciones & $7(6.9)$ \\
Diabetes mellitus y/o complicaciones de la diabetes & $6(5.9)$ \\
Enfermedades reumatológicas e inmunológicas & $4(3.9)$ \\
Estados encefalopáticos y síncope & $4(3.9)$ \\
Patología renal & $3(2.9)$ \\
Infecciones parasitarias/tropicales & $2(2)$ \\
\hline
\end{tabular}

Tabla 3. Clasificación de pacientes según el riesgo de TEV.

\begin{tabular}{|l|r|}
\hline Clasificación por riesgo, $\mathbf{n}(\%)$ & \\
Alto riesgo para TEV & $70(68.6)$ \\
Bajo riesgo para TEV & $32(31.3)$ \\
\hline Clasificación por diagnóstico y/o puntaje, $\mathbf{n}(\%)$ & \\
Alto riesgo para TEV únicamente por puntaje & $8(11,4)$ \\
Alto riesgo para TEV únicamente por diagnóstico & $15(21.4)$ \\
Alto riesgo para TEV por puntaje y diagnóstico & $47(67.2)$ \\
\hline
\end{tabular}

para recibir heparinas no se formuló profilaxis mecánica en ningún caso. La Tabla 4 muestra un consolidado sobre los errores implicados en la mala formulación de la profilaxis antitrombótica.

El medicamento de uso más frecuente fue enoxaparina en $84 \%$ de los casos, seguida por la heparina no fraccionada en $14.6 \%$ y nadroparina con $1.3 \%$. No se utilizó profilaxis mecánica en ningún paciente.

Tabla 4. Adherencia a la tromboprofilaxis y causas de profilaxis inadecuada.

\begin{tabular}{|l|c|}
\hline $\begin{array}{l}\text { Adherencia a la tromboprofilaxis. } \\
\text { Profilaxis adecuada }\end{array}$ & $63(61.8)$ \\
Profilaxis inadecuada & $39(38.2)$ \\
\hline Causas de profilaxis inadecuada & $18(46.1)$ \\
Formulación en bajo riesgo de TVE* & $12(30.7)$ \\
Omisión & $4(10.2)$ \\
Mala elección del fármaco & $3(7.6)$ \\
Falta de formulación de profilaxis mecánica $\dagger$ & $1(2.5)$ \\
Profilaxis farmacológica con alto riesgo de sangrado & $1(2.5)$ \\
Dosis inadecuada & \\
\hline * Uno de estos pacientes tuvo además alto riesgo de sangrado \\
$\dagger$ Pacientes de alto riesgo con contraindicación para profilaxis farmacológica
\end{tabular}




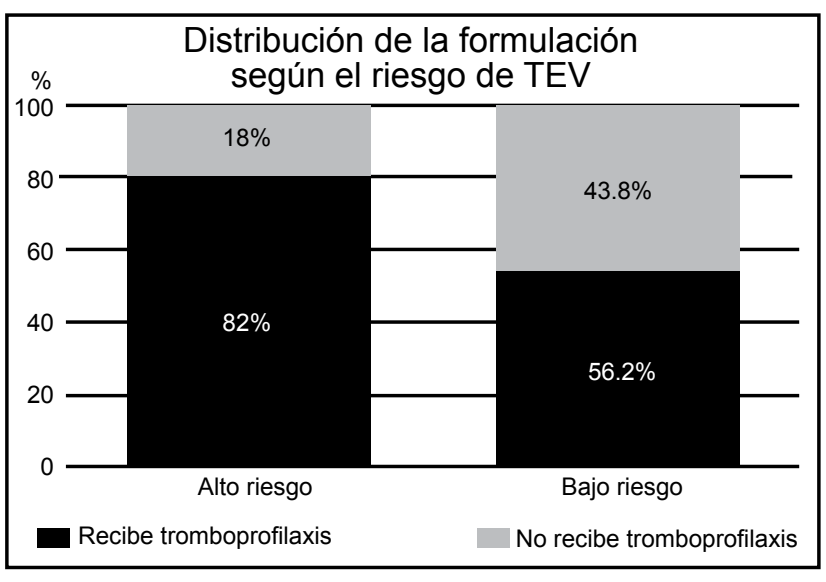

Figura 1. Porcentaje de formulación de tromboprofilaxis según el riesgo de tromboembolia venosa.

\section{Comparación con resultados anteriores en la institución}

Entre los pacientes con indicación de profilaxis farmacológica sin contraindicaciones, se formularon los fármacos en $82 \%$ ( 88 y $92 \%$ para la primera y segunda medición). Entre los pacientes de bajo riesgo se formuló profilaxis en $56.2 \%$ (86 y $50 \%$ en la primera y segunda medición).

\section{Discusión}

En nuestro hospital se lleva a cabo un programa de mejoramiento continuado en el tema de la tromboprofilaxis liderado por el servicio de hematología. Este inició con un proceso diagnóstico durante el año 2009, en el cual se identificaron posibles barreras que habían en cada servicio para formular una profilaxis adecuada, posteriormente se realizó una medición inicial en ese año que sirvió como base para conocer el estado de la tromboprofilaxis en la institución y se diseñaron varias estrategias de implementación de guías aplicadas desde 2010, en los pacientes de hospitalización general. Una segunda medición se hace entre 2011 y 2012 , en la cual se evidenció una mejoría importante de la tromboprofilaxis, principalmente en la disminución del error por omisión, sin embargo en pacientes de bajo riesgo aumentó el uso de la profilaxis. En los pacientes de medicina interna evaluados, el impacto de la guía fue mayor en la disminución de la formulación en pacientes de bajo riesgo (Solano $\mathrm{MH}$, comunicación personal, 08 de febrero de 2014).

La evaluación realizada en el presente trabajo corresponde a una tercera medición, en la cual se documenta que la mejoría obtenida en la segunda medición disminuyó levemente. En general se observó una disminución leve en el uso adecuado de la tromboprofilaxis. Las diferencias encontradas en los resultados de las mediciones pueden tener varias explicaciones. En primer lugar la segunda medición refleja la aplicación de una estrategia de difusión, motivo por el cual era de esperarse una tasa de adherencia mayor. En segundo lugar, el hospital al ser universitario está constituido en su mayoría por personal médico en formación sujeto a recambio frecuente en los servicios, lo cual influye directamente en la formulación de los pacientes. En tercer lugar las mediciones tuvieron algunas diferencias como el número de pacientes evaluados y la forma de recolección de los mismos que puede explicar en parte las diferencias encontradas.

La alta rotación del personal encargado de la formulación es una limitante importante en la aplicación de estas estrategias, ya que si no se hacen actividades educativas en este personal dirigidas al tema de la tromboprofilaxis es posible que la estrategia pueda perder su efectividad con el tiempo. Adicionalmente, para la fecha de evaluación de esta investigación ya se habían publicado otros consensos internacionales con directrices que difieren en algunos puntos con la guía institucional (12), lo que también pudo afectar la decisión a un personal "nuevo" en la institución.

Un tema importante tiene que ver con la estrategia utilizada para la implementación de guías. Esto es fundamental a la hora de evaluar una intervención, de hecho la guía ACCP (American College of Chest Physicians) publicada en 2004, tuvo un capítulo dedicado a las estrategias de difusión de guías para la tromboprofilaxis (13), en donde se mencionan las ventajas y desventajas de cada una de ellas. En general las estrategias de difusión "activas" como los sistemas de soporte de decisión computarizados y las alertas electrónicas están mejor documentadas en cuanto a su efectividad $(10,14)$. En nuestro caso se aplicaron estrategias de difusión denominadas "pasivas" como reuniones académicas, manuales de bolsillo y ayudas gráficas como afiches, lo cual tiene limitaciones en el beneficio que pueda lograr a largo plazo.

Otro punto por considerar es la dificultad que tiene evaluar y calificar como adecuada o errónea la estrategia antitrombótica. Al respecto, la forma de estratificación de riesgo trombótico es fundamental y no hay una forma única de hacerlo en el paciente médico. En general, existen estrategias basadas en el diagnóstico y otras individuales desarrolladas como "modelos de evaluación de riesgo" o "RAM". Cada forma tiene ventajas y desventajas, sin haber un modelo que logre estratificar con exactitud a los pacientes, algunos han sido dirigidos a poblaciones específicas como pacientes oncológicos y otros no han sido validadas (12, 15-19). Las últimas guías de la práctica clínica ACCP le da mayor valor a los modelos de evaluación de riesgo por puntaje, dentro de los cuales propone la escala de Padua (20). Sin embargo, también tienen algunas limitaciones de las mencionadas y se puede adjudicar bajas puntuaciones en contextos como la enfermedad cardiaca, pulmonar e infecciones agudas en donde normalmente se administra profilaxis. En nuestro caso se utilizaron dos formas para estratificar el riesgo, lo cual si bien es cierto no es una recomendación generalizada, también es cierto que no hay un consenso en este tema.

La formulación adecuada de profilaxis vista en este estudio no se aleja de la realidad expuesta en otras evaluaciones internacionales como en el estudio IMPROVE en donde el uso de profilaxis adecuada estuvo entre 62 y 64\%(2). De 
hecho, muchos reportes documentan profilaxis adecuada en porcentajes inferiores a 50\% $(3,21,22)$. En Colombia este es uno de los temas que ha sido sujeto a investigación y que tiene información que nos permite vislumbrar nuestra realidad. En 1996 se publicó el estudio nacional sobre TEV en donde $51 \%$ de los pacientes tenían al menos un factor de riesgo para TEV y no se administró profilaxis en $28 \%$ (23). También nuestro país participó en los registros internacionales IMPROVE y ENDORSE. En el primero, del cual nuestra institución fue partícipe se formuló tromboprofilaxis en $76 \%$ de los pacientes candidatos a recibirla. Este porcentaje fue mayor que el promedio a nivel mundial (60\%); sin embargo, también se documentó que en nuestros hospitales el error de formulación en pacientes de bajo riesgo fue mayor que el promedio del resto de países evaluados (24). En el estudio ENDORSE, la profilaxis en pacientes médicos con riesgo de TEV fue $63.7 \%$. Si bien los resultados fueron un poco mejores que el promedio encontrado, también documenta que hay mucho por hacer en este tema (25).

Hay datos de instituciones de nuestro país en donde se evaluó la profilaxis en pacientes de hospitalización general y en el contexto del cuidado crítico (26-28). Por lo que, si bien es cierto, se tiene más o menos documentada la realidad en este tema, y también conocemos de algunas estrategias hospitalarias para mejorar el uso de la tromboprofilaxis, impulsadas principalmente por la industria farmacéutica, hasta el momento no conocemos resultados de estudios publicados que hayan evaluado estrategias para mejorar esta situación en nuestro medio. Es importante que en este tema se dé un paso hacia adelante en la evaluación de diversas estrategias de implementación de la tromboprofilaxis. Las estrategias que han demostrado ser benéficas en "otras latitudes" no necesariamente sirven de forma "automática" en nuestros hospitales, las realidades de ellos no son las mismas que las de nosotros. Por lo tanto, se deben diseñar estudios o presentar resultados de las experiencias de investigación en este sentido para también aprender de nosotros mismos y poder brindar una mejor atención a nuestros pacientes.

Las principales limitaciones de este estudio se relacionan con los sesgos inherentes de un diseño retrospectivo de corte transversal. Sólo evaluamos el uso de la tromboprofilaxis a las 48 horas, que si bien es un buen punto de referencia, no necesariamente refleja la tromboprofilaxis durante toda la hospitalización. Este enfoque no tiene en cuenta posibles variaciones en la condición de los pacientes durante la hospitalización, aunque varios estudios ya nombrados se diseñan bajo este formato. Otra posible fuente de error es el sesgo información, ya que los datos son obtenidos de manera retrospectiva según lo consignado en la historia clínica, en la cual se corre el riesgo de omitir posible información de interés para el estudio.

Como fortalezas de nuestro estudio destacamos un tamaño de muestra suficiente para disminuir el error en las estimaciones, la selección aleatoria de las historias clínicas para la recolección de los datos y el poder contar con información previa, ya que esto permitió realizar una comparación en tres momentos diferentes. Debe señalarse que si bien existen dificultades al momento de calificar como adecuada o errónea la tromboprofilaxis, se aseguró la realización de un consenso mínimo de tres investigadores con dominio del tema para casos de difícil decisión. Otra fortaleza fue la evaluación integral que se realizó de la tromboprofilaxis, en la cual se pudo discriminar los tipos de errores más frecuentes, lo cual es importante a la hora de planificar las estrategias de implementar guías.

\section{Conclusiones}

El escenario de tromboprofilaxis en nuestro hospital visto en estos resultados no fue tan distinto al de otros estudios, las intervenciones realizadas en años previos fue benéfica en algún aspecto, sin embargo no son suficientes para garantizar en el tiempo una adecuada formulación de la profilaxis. Se plantea que es necesario complementar las estrategias utilizadas preferiblemente con algunas de carácter activo, teniendo en cuenta las particularidades de nuestra institución, en donde el mayor error es la formulación en pacientes de bajo riesgo y una dificultad mayor es la alta rotación de personal. También es importante incentivar el uso de medidas mecánicas antitrombóticas en el paciente con contraindicaciones de profilaxis medicamentosa. Este estudio plantea además la necesidad de instaurar estrategias ajustadas a nuestro medio para que la tromboprofilaxis sea tema de interés e interiorización diaria del médico que labora en los hospitales de nuestro país.

\section{Agradecimientos}

A la doctora Lizet Moreno por su valioso aporte metodológico, a la doctora María Helena Solano Trujillo por facilitar importante información relevante para el estudio y al departamento de estadística del hospital.

Ninguno

\section{Conflicto de interés}

\section{Referencias}

1. Douketis JD. Prevention of venous thromboembolism in hospitalized medical patients: addressing some practical questions. Current opinion in pulmonary medicine. 2008; 14(5): 381-8. Epub 2008/07/31.

2. Tapson VF, Decousus H, Pini M, Chong BH, Froehlich JB, Monreal M, et al. Venous thromboembolism prophylaxis in acutely ill hospitalized medical patients: findings from the International Medical Prevention Registry on Venous Thromboembolism. Chest. 2007; 132(3): 936-45. Epub 2007/06/19.

3. Cohen AT, Tapson VF, Bergmann JF, Goldhaber SZ, Kakkar AK, Deslandes $\mathbf{B}$, et al. Venous thromboembolism risk and prophylaxis in the acute hospital care setting (ENDORSE study): a multinational cross-sectional study. Lancet. 2008; 371(9610): 387-94. Epub 2008/02/05.

4. Tooher R, Middleton P, Pham C, Fitridge R, Rowe S, Babidge W, et al. A systematic review of strategies to improve prophylaxis for venous thromboembolism in hospitals. Annals of surgery. 2005; 241(3): 397-415. Epub 2005/02/25.

5. The National Quality Forum. National Voluntary Consensus Standards for Prevention and Care of Venous Thromboembolism: Additional Performance Measures. United States Of America2008 [cited 2014 Marzo 28]; Available from: http://www. qualityforum.org/Projects/s-z/VTE_Phase_II_(2008)29/VTE_Phase_II.aspx

6. Hill J, Treasure T. Reducing the risk of venous thromboembolism in patients admitted to hospital: summary of NICE guidance. BMJ. 2010; 340: c95. Epub 2010/01/29

7. Safer Healthcar Now. Venous Thromboembolism prevention. Canada2012 [updated Mayo 2012; cited 2014 Marzo 28]; Available from: http://www.safer- 
healthcarenow.ca/EN/Interventions/vte/Pages/default.aspx

8. Abuabara J, Acero R, Archila PE, Alí A, Bermúdez M, Bohórquez R, et al. II Consenso Colombiano de Tromboembolismo venoso. Acta Med Colomb. 2005;30(supl.3):175-252.

9. Solano MH. Tromboprofilaxis en el Hospital San José. Sociedad de Cirugía de Bogotá Hospital San José: Guias de práctica clínica Hematología; 2013.

10. Durieux P, Nizard R, Ravaud P, Mounier N, Lepage E. A clinical decision support system for prevention of venous thromboembolism: effect on physician behavior. JAMA 2000; 283(21): 2816-21. Epub 2000/06/06.

11. Stevens SM, Douketis JD. Deep vein thrombosis prophylaxis in hospitalized medical patients: current recommendations, general rates of implementation, and initiatives for improvement. Clinics in chest medicine. 2010; 31(4): 675-89. Epub 2010/11/05

12. Kahn SR, Lim W, Dunn AS, Cushman M, Dentali F, Akl EA, et al. Prevention of VTE in nonsurgical patients: Antithrombotic Therapy and Prevention of Thrombosis, 9th ed: American College of Chest Physicians Evidence-Based Clinical Practice Guidelines. Chest. 2012;141 (2Suppl): e195S-226S. Epub 2012/02/15.

13. Schunemann HJ, Cook D, Grimshaw J, Liberati A, Heffner J, Tapson V, et al. Antithrombotic and thrombolytic therapy: from evidence to application: the Seventh ACCP Conference on Antithrombotic and Thrombolytic Therapy. Chest. 2004; 126(3 Suppl): 688S-96S. Epub 2004/09/24.

14. Kucher N, Koo S, Quiroz R, Cooper JM, Paterno MD, Soukonnikov B, et al. Electronic alerts to prevent venous thromboembolism among hospitalized patients. The New Eng J Med. 2005; 352(10): 969-77. Epub 2005/03/11.

15. Spyropoulos AC. Risk assessment of venous thromboembolism in hospitalized medical patients. Current opinion in pulmonary medicine. 2010; 16(5): 419-25. Epub 2010/07/02.

16. Caprini JA, Arcelus JI, Reyna JJ. Effective risk stratification of surgical and nonsurgical patients for venous thromboembolic disease. Seminars in hematology. 2001; 38(2 Suppl 5): 12-9. Epub 2001/07/13.

17. Cohen AT, Alikhan R, Arcelus JI, Bergmann JF, Haas S, Merli GJ, et al. Assessment of venous thromboembolism risk and the benefits of thromboprophylaxis in medical patients. Thrombosis and haemostasis. 2005; 94(4): 750-9. Epub 2005/11/08

18. Lutz L HS, Hach-Wunderle V. Venous thromboembolism in internal medicine: risk assessment and pharmaceutical prophylaxis. Med Welt. 2002; 53: 231-4.
19. Laporte S, Mismetti P. Epidemiology of thrombotic risk factors: the difficulty in using clinical trials to develop a risk assessment model. Critical care medicine. 2010; 38(2 Suppl): S10-7. Epub 2010/01/30.

20. Barbar S, Noventa F, Rossetto V, Ferrari A, Brandolin B, Perlati M, et al. A risk assessment model for the identification of hospitalized medical patients at risk for venous thromboembolism: the Padua Prediction Score. Journal of thrombosis and haemostasis : JTH. 2010; 8(11): 2450-7. Epub 2010/08/27.

21. Amin A, Stemkowski S, Lin J, Yang G. Thromboprophylaxis rates in US medical centers: success or failure? Journal of thrombosis and haemostasis: JTH. 2007; 5(8): 1610-6. Epub 2007/08/01

22. Yu HT, Dylan ML, Lin J, Dubois RW. Hospitals' compliance with prophylaxis guidelines for venous thromboembolism. American journal of health-system pharmacy. AJHP: Official journal of the American Society of Health-System Pharmacists. 2007; 64(1): 69-76. Epub 2006/12/26.

23. Dennis R, de Arboleda MN, Rodríguez MN, Salazar MS, Posada PS. Estudio nacional sobre tromboembolismo venoso en población hospitalaria en Colombia. Diferencias entre ciudades y especialidades. Acta Med Col. 1996; 21(2): 55-63.

24. Dennis RJ, Acevedo JR, Restrepo HF, Hernández JI, Rivas E, Sabogal JE. ¿Es apropiada la profilaxis actual del tromboembolismo venoso en pacientes médicos?: Evaluación de desenlaces colombianos en el Registro Internacional sobre Prevención Médica del TEV (IMPROVE). Acta Medica Colombiana. 2009; 34: 11-6.

25. Dennis RJ, Roa JH, Villadiego J, Mendez F, Vieda E, Restrepo H. Profilaxis de la tromboembolia venosa en pacientes colombianos de tratamiento medico o quirurgico: resultados para Colombia del estudio ENDORSE. Biomedica: revista del Instituto Nacional de Salud. 2011; 31(2): 200-8. Epub 2011/12/14.

26. Velásquez JC, Herrán S, Mera I. Uso de tromboprofilaxis en el paciente hospitalizado en la Clínica del Country Un análisis de la adherencia a la guía institucional. Acta Medica Colombiana. 2010; 35: 8-14.

27. Diaztagle JJ, Melo CE, Sprockel JJ, Cruz LE, Navarrete J, Pérez D, et al. Calidad de la profilaxis farmacológica antitrombótica en pacientes hospitalizados en la unidad de cuidados intensivos del Hospital El Tunal de Bogotá Acta Colombiana de Cuidado Intensivo. 2010; 10(4): 236-43.

28. Solano J, Londoño D. Evaluacion de escalas para evaluar el riesgo de tromboembolismo venoso en la UCI en el Hospital Universitario San Ignacio. Acta Medica Colombiana. 2006; 31(3): 360 . 